\title{
Plantio de aveia-preta na região de São Mateus no estado do Espirito Santo, Brasil
}

\section{Oat planting in the region of São Mateus in the State of Espirito Santo, Brazil}

\author{
Dione Pereira Cardoso ${ }^{1}$, Lucas Rodrigues Nicole ${ }^{2}$, Gleison Oliosi ${ }^{3}$, Fábio Ribeiro Pires $^{4}$
}

Resumo: Dentre as cultivares de aveia-preta, optou-se pela cultivar Embrapa 29 (Garoa) por ser uma espécie de boa produção de massa e adaptada às condições da região Sudeste, porém não há estudo do cultivo da aveia-preta para a região norte do Espírito Santo. Portanto, avaliar o crescimento e desenvolvimento da Avena strigosa cultivar Embrapa 29 (Garoa) nas condições edafoclimáticas do norte do ES, torna-se de suma importância para região, visto que essa propicia várias melhorias na qualidade do solo. A área onde foi instalado o experimento, pertence à Universidade Federal do Espírito Santo, Centro Universitário Norte do Espírito Santo-CEUNES, câmpus de São Mateus - ES. A semeadura foi realizada com uma máquina de plantio direto em sulcos espaçados de $0,30 \mathrm{~m}$. Não realizou a correção da acidez do solo e nem aplicações de adubos minerais e orgânicos. Mesmo com o plantio tardio, a aveia-preta teve um bom desenvolvimento nas condições edafoclimáticas da região norte do ES. Este, possivelmente, foi beneficiado pela textura, visto que não há nenhum impedimento para o sistema radicular da planta. A aveia-preta desenvolve bem nas condições edafoclimáticas da região de São Mateus-ES, principalmente, sobre um Argiloso Amarelo de textura do tipo areia-franca.

Palavras-chaves: Aveia strigosa Schreb., cultivar Embrapa 29, condições edafoclimáticas, Argissolo Amarelo distrófico.

\begin{abstract}
Among the cultivars of oat, it was decided to Embrapa 29 (Garoa) to be a good kind of mass production and adapted to the conditions in the Southeast. But there is no study of the cultivation of oat to the northern region of the Holy Spirit. Therefore, assessing the growth and development of Avena strigosa Embrapa 29 (Garoa) at conditions of northern ES, it is of paramount importance for the region, since this provides a number of improvements in soil quality. The area where the experiment was conducted, belongs to the Federal University of Espirito Santo, University Center North-CEUNES Holy Spirit campus of St. Matthew - ES. Sowing was carried out with a tillage machine in spaced grooves of $0.30 \mathrm{~m}$. Did not make the correct soil acidity and, mineral and organic fertilizers applications. Even with the late planting, oat had a good development in the environmental conditions of the northern region ES. This possibly benefited from the texture, since there is no impediment to the root system of the plant. The black oats grow well in soil and climatic conditions of São Mateus-ES region, mainly on a Yellow Argillaceous type of texture sand-franca.
\end{abstract}

Key words: Aveia strigosa Schreb., cultivate Embrapa 29, edaphoclimatic conditions, distrophic yellow Argisol.

\footnotetext{
*Autor para correspondência

Recebido para publicação em 04/11/2015; aprovado em 12/12/2015

${ }^{1}$ Bolsista de Profix da Capes, Centro Universitário Norte do Espírito Santo-CEUNES, Universidade Federal do Espírito Santo-UFES, São Mateus-ES, (27) 9.9986-6293, E-mail: cardoso.dione@gmail.com

${ }^{2}$ Mestrando em Agricultura Tropical, Centro Universitário Norte do Espírito Santo-CEUNES, Universidade Federal do Espírito Santo-UFES, São Mateus-ES.

E-mail: lukssnicoli@hotmail.com

${ }^{3}$ Mestrando em Agricultura Tropical, Centro Universitário Norte do Espírito Santo-CEUNES, Universidade Federal do Espírito Santo-UFES, São Mateus-ES

E-mail: gleison.oliosi@ hotmail.com

${ }^{4}$ Professor Associado I, Centro Universitário Norte do Espírito Santo-CEUNES, Universidade Federal do Espírito Santo-UFES, São Mateus-ES. E-mail: pires.fr@gmail.com
} 


\section{INTRODUÇÃO}

Em 1771, Johann Christian Daniel Von Schreber descreveu a espécie aveia-preta. Sua classificação taxonômica é a seguinte: classe Equisetopsida; subclasse Magnoliidae; super ordem Liliane; ordem Poales; família Poaceae; e gênero Avena L. Atualmente, há 95 espécies do gênero Avena sp (MISSOURI BOTANICAL GARDEN, 2015). No Brasil, há duas espécies que são muito cultivadas, sendo Avena sativa L. (aveia-branca) e Avena strigosa Schreb (aveia-preta).

Esta é uma planta anual, que desenvolve bem no outono, inverno e início da primavera, sendo de acordo com Calegari et al. (1993) muito difundida na região Sul do país, mas viável nas regiões norte de São Paulo, Mato Grosso do Sul e Minas Gerais. De acordo com Wutke et al. (2014), são espécies com desenvolvimento favorecido por temperaturas amenas e restrição de chuvas, com ciclo encerrado ao final do inverno, no máximo até o início da primavera.

Dentre as cultivares de aveia-preta, optou-se pela cultivar Embrapa 29 (Garoa) por ser uma espécie de boa produção de massa e adaptada às condiçõos da região Sudeste. A aveiapreta, de acordo com Wutke et al. (2014), são anuais, eretas, com sistema radicular fasciculado e rendimento médio de fitomassa verde correspondente a 50 t ha-1. Ferolla et al. (2007), observaram que a melhor época para plantio desta cultivar de aveia-preta no norte do Rio de Janeiro é de maio/junho no sistema de corte.

Em sistemas de sequeiro, Sousa \& Lobato (2004), recomenta elevar a saturação por bases a $50 \%$ para o plantio de aveia-preta. De acordo como mesmos, para a adubação de semeadura, as dosagens adequadas de $\mathrm{N}, \mathrm{P}_{2} \mathrm{O}_{5}$ e $\mathrm{K}_{2} \mathrm{O}$ para um rendimento de $3 \mathrm{t} \mathrm{ha}^{-1}$ seriam de $30 \mathrm{Kg} \mathrm{ha}^{-1}, 70 \mathrm{~kg} \mathrm{ha}^{-1}$ e 40 $\mathrm{kg} \mathrm{ha}{ }^{-1}$, respectivamente. Schuch et al. (1999), observaram que o comprimento do sistema radicular é o parâmetro mais adequado para avaliações de diferenças em vigor. Como plantas de cobertura ou adubação verde, o manejo da fitomassa deve ser realizado na fase do grão leitoso, correspondendo ao período entre 120 a 140 dias após a semeadura (CALEGARI et al., 1993). Tem elevado efeito alelopático em muitas plantas invasoras, sendo reduzidos os custos de manutenção com capinas ou herbicidas (WUTKE et al., 2014). De acordo com Burle et al. (2006), este efeito permanece mesmo depois da sua colheita.

Não há estudo do cultivo da aveia-preta para a região norte do Espírito Santo. Portanto, avaliar o crescimento e desenvolvimento da Avena strigosa cultivar Embrapa 29 (Garoa) nas condições edafoclimáticas do norte do ES, torna- se de suma importância para região, visto que essa propicia várias melhorias na qualidade do solo.

\section{MATERIAL E MÉTODOS}

A área onde foi instalado o experimento, pertence à Universidade Federal do Espírito Santo, Centro Universitário Norte do Espírito Santo-CEUNES, câmpus de São Mateus ES. A área experimental apresenta coordenadas geográficas correspondentes a $18^{\circ} 40^{\prime} 26,26^{\prime \prime} \mathrm{S}$ e $39^{\circ} 51^{\prime} 25,91^{\prime \prime} \mathrm{W}$, com altitude de $36 \mathrm{~m}$. A região de São Mateus apresenta clima do tipo Aw, de acordo com a classificação de Köppen, clima tropical com estação seca de inverno (PEEL et al. 2007).

O solo no local do experimento é classificado como Argissolo Vermelho-Amarelo Distrófico (EMBRAPA, 2013), com relevo plano e textura do tipo areia-franca.

A semeadura foi realizada com uma máquina de plantio direto em sulcos espaçados de 0,30 m, no dia 03/06/2015. Antes do plantio da aveia-preta foi realizado o preparo convencional do solo, sendo uma aração, duas gradagens e uma grade niveladora. Não realizou a correção da acidez do solo e nem aplicações de adubos minerais e orgânicos.

A área foi cultivada com a espécie do gênero Eucalyptus spp. e, após a destoca, ocorreu o cultivo de feijão-de-porco (Canavalia ensiformis) e crotalária (Crotalaria juncea). $\mathrm{Na}$ área experimental, antes do plantio da aveia-preta, predominava a espécie Brachiaria sp.

A análise química foi determinada, conforme Embrapa (1997): o pH em água, os macronutrientes (P, P-rem, $\mathrm{K}^{+}, \mathrm{Ca}^{2+}$ e $\left.\mathrm{Mg}^{2+}\right)$, os parâmetros de fertilidade $\left(\mathrm{Al}^{3+}, \mathrm{H}^{+}+\mathrm{Al}^{3+}, \mathrm{SB}, \mathrm{t}\right.$, $\mathrm{T}, \mathrm{V}$ e m) e a matéria orgânica. Em relação à análise física determinou-se a análise granulométrica pelo método da pipeta (DAY, 1965).

\section{RESULTADOS E DISCUSSÃO}

O solo da área experimental é um classificado quanto à sua fertilidade como distrófico (Tabela 1), ou seja, a saturação por bases está abaixo de $50 \%(47,2 \%)$, sendo a classe textural denominada de areia-franca (Tabela 2). De acordo com a classificação da Embrapa (2013), este é classificado como Argiloso Amarelo. A aveia-preta é mais rústica, desenvolvendo bem em solo pouco fértil (WUTKER et al., 2014). A aveia-preta, de acordo com Burle et al. (2006), não é muito exigente quanto à fertilidade dos solos, mas atinge maiores produções em solos férteis, com alto teor de matéria orgânica, permeáveis e bem drenado.

Tabela 1 - Análise química de uma Argissolo Amarelo localizado na Fazenda Experimental, Centro Universitário Norte do Espírito Santo-Ceunes, câmpus de São Mateus-ES.

\begin{tabular}{|c|c|c|c|c|c|}
\hline Atributos & S. I. & Valores & Atributos & S. I. & Valores \\
\hline$\overline{\mathrm{pH}}$ & $\left(\mathrm{H}_{2} \mathrm{O}\right)$ & 5,4 & $\mathrm{Al}^{3+}$ & $\left(\mathrm{cmol}_{\mathrm{c}} \mathrm{dm}^{-3}\right)$ & 0,1 \\
\hline $\mathrm{P}$ & $\left(\mathrm{mg} \mathrm{dm}^{-3}\right)$ & 4,1 & $\mathrm{H}^{+}+\mathrm{Al}^{3+}$ & $\left(\mathrm{cmol}_{\mathrm{c}} \mathrm{dm}^{-3}\right)$ & 1,6 \\
\hline $\mathrm{K}^{+}$ & $\left(\mathrm{mg} \mathrm{dm}^{-3}\right)$ & 29,0 & SB & $\left(\mathrm{cmol}_{\mathrm{c}} \mathrm{dm}^{-3}\right)$ & 1,4 \\
\hline $\mathrm{Ca}^{2+}$ & $\left(\mathrm{cmol}_{\mathrm{c}} \mathrm{dm}^{-3}\right)$ & 1,0 & $\mathrm{t}$ & $\left(\mathrm{cmol}_{\mathrm{c}} \mathrm{dm}^{-3}\right)$ & 1,5 \\
\hline $\mathrm{Mg}^{2+}$ & $\left(\mathrm{cmol}_{\mathrm{c}} \mathrm{dm}^{-3}\right)$ & 0,4 & $\mathrm{~T}$ & $\left(\mathrm{cmol}_{\mathrm{c}} \mathrm{dm}^{-3}\right)$ & 3,0 \\
\hline $\mathrm{Na}^{+}$ & $\left(\mathrm{mg} \mathrm{dm}^{-3}\right)$ & 5,5 & Sat. Ca & $(\%)$ & 31,8 \\
\hline $\mathrm{Fe}$ & $\left(\mathrm{mg} \mathrm{dm}^{-3}\right)$ & 93,8 & Sat. Mg & $(\%)$ & 12,0 \\
\hline $\mathrm{Mn}$ & $\left(\mathrm{mg} \mathrm{dm}^{-3}\right)$ & 3,9 & Sat. K & $(\%)$ & 2,6 \\
\hline $\mathrm{Zn}$ & $\left(\mathrm{mg} \mathrm{dm}^{-3}\right)$ & 0,4 & $\mathrm{~m}$ & $(\%)$ & 6,9 \\
\hline $\mathrm{Cu}$ & $\left(\mathrm{mg} \mathrm{dm}^{-3}\right)$ & 0,3 & $\mathrm{~V}$ & $(\%)$ & 47,2 \\
\hline MO & $\left(\right.$ dag kg $\left.^{-1}\right)$ & 1,9 & & & \\
\hline
\end{tabular}


Tabela 2 - Análise granulométrica do solo.

\begin{tabular}{|c|c|c|c|}
\hline Horizontes & Areia & Silte & Argila \\
\hline $\mathrm{A}(0-20 \mathrm{~cm})$ & 800 & $\begin{array}{c}-\mathrm{g} \mathrm{kg}^{-1} \\
105\end{array}$ & 95 \\
\hline
\end{tabular}

A aveia-preta desenvolveu bem nesse tipo de solo, porque de acordo com as características químicas do solo, este apresenta um $\mathrm{pH}$ próximo ao ideal para a cultura, que seria de 5,0 a 7,0. Consequentemente, há um baixo valor de alumínio trocável. O teor de matéria orgânica é baixo, porém, é o valor que predomina em boa parte dos solos brasileiros.

Conforme as Figuras 1 e 2, durante a condução do experimento observaram-se as seguintes as condições climáticas: de $19,7{ }^{\circ} \mathrm{C}$ a $25,0{ }^{\circ} \mathrm{C}$ (temperatura instantânea), de $20,4{ }^{\circ} \mathrm{C}$ a $25,5{ }^{\circ} \mathrm{C}$ (temperatura máxima), de $19,1^{\circ} \mathrm{C}$ a $24,6{ }^{\circ} \mathrm{C}$ (temperatura mínima), de $77,4 \%$ a $95,2 \%$ (umidade instantânea), de 79,5 \% a 96,8 \% (umidade máxima), de 74,5 $\%$ a $93,1 \%$ (umidade mínima), de $17,0{ }^{\circ} \mathrm{C}$ a $21,5{ }^{\circ} \mathrm{C}$ (ponto de orvalho instantâneo), de $17,6{ }^{\circ} \mathrm{C}$ a $21,8{ }^{\circ} \mathrm{C}$ (ponto de orvalho máximo), de $16,5{ }^{\circ} \mathrm{C}$ a $21,2{ }^{\circ} \mathrm{C}$ (ponto de orvalho mínimo), de $1010,0 \mathrm{hPa}$ a $1020,5 \mathrm{hPa}$ (pressão atmosférica instantânea), de 1010,2 hPa a 1020,7 hPa (pressão atmosférica máxima), de $1009,7 \mathrm{hPa}$ a $1020,3 \mathrm{hPa}$ (pressão atmosférica mínima), de $1,3 \mathrm{~m} / \mathrm{s}$ a $4,4 \mathrm{~m} / \mathrm{s}$ (velocidade do vento), de $41,2^{\circ}$ a $242,0^{\circ}$ (direção do vento), de $2,8 \mathrm{~m} / \mathrm{s}$ a 8,6 $\mathrm{m} / \mathrm{s}$ (rajada de vento), de $232,0 \mathrm{KJ} / \mathrm{m}^{2}$ a $1245,0 \mathrm{KJ} / \mathrm{m}^{2}$ (radiação solar) e de 0,2 $\mathrm{mm}$ a $11,4 \mathrm{~mm}$ (precipitação). Estas condições são diferentes das condições edafoclimáticas da região Sul do Brasil, onde predomina o cultivo da aveia-preta.

Figura 1 - As condições climáticas referentes à temperatura, umidade, pressão atmosférica e ponto de orvalho, durante o período de 03/07/2015 a 10/09/2015, correspondente a 70 dias.
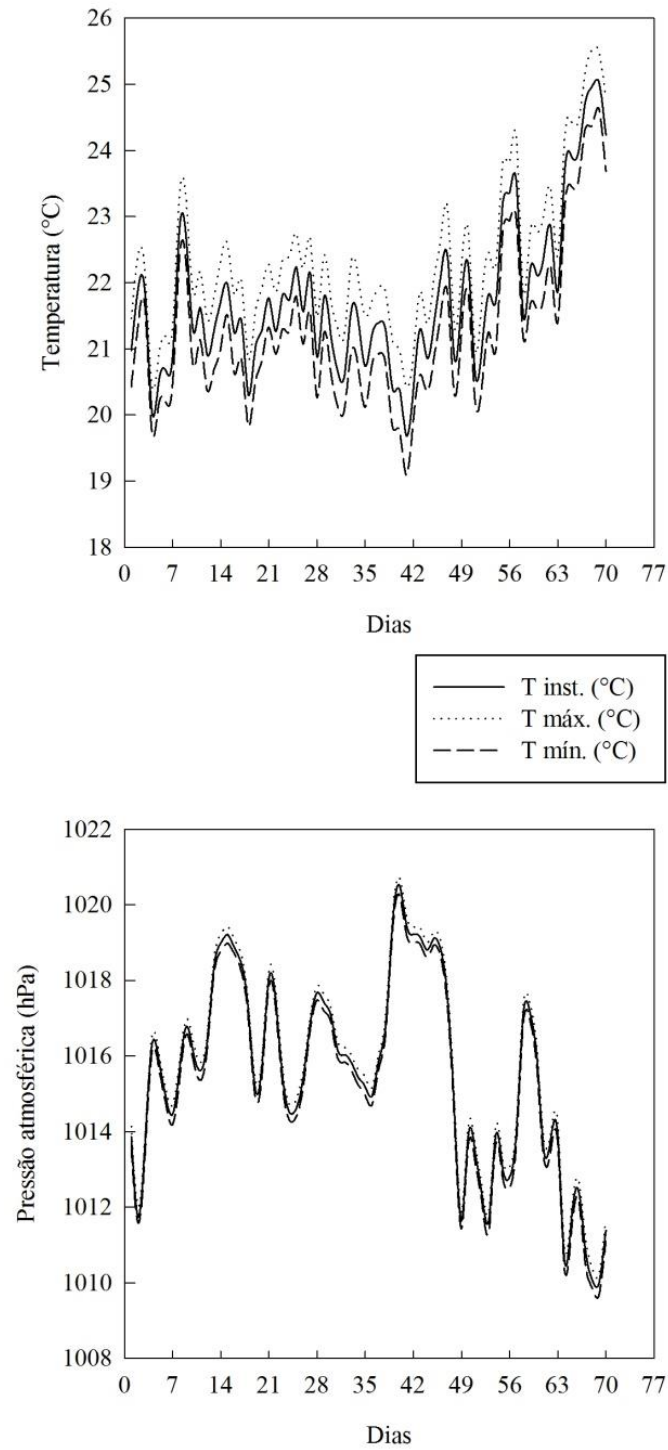

Dias

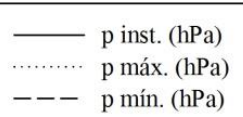

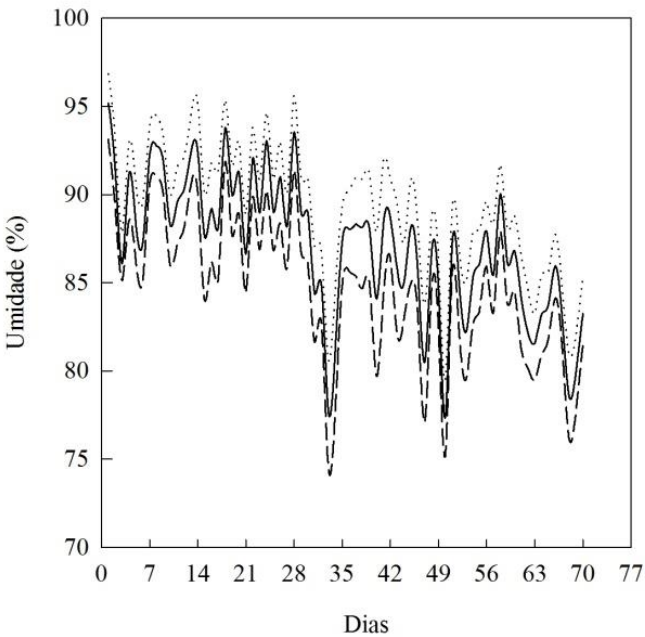
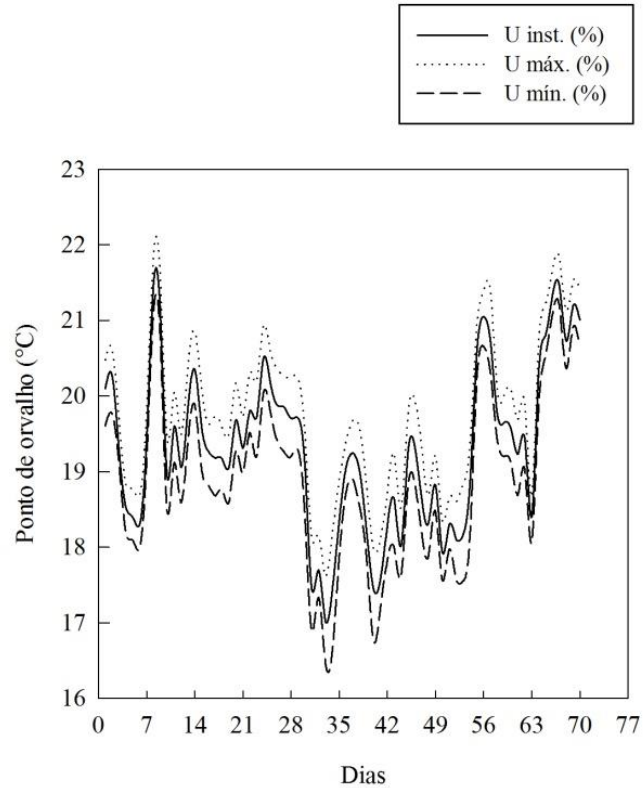

Dias

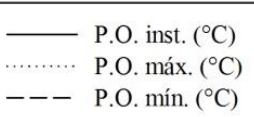


Figura 2 - As condições climáticas referentes à velocidade do vento, rajada de vento, direção do vento, precipitação e radiação solar, durante o período de 03/07/2015 a 10/09/2015, correspondente a 70 dias.
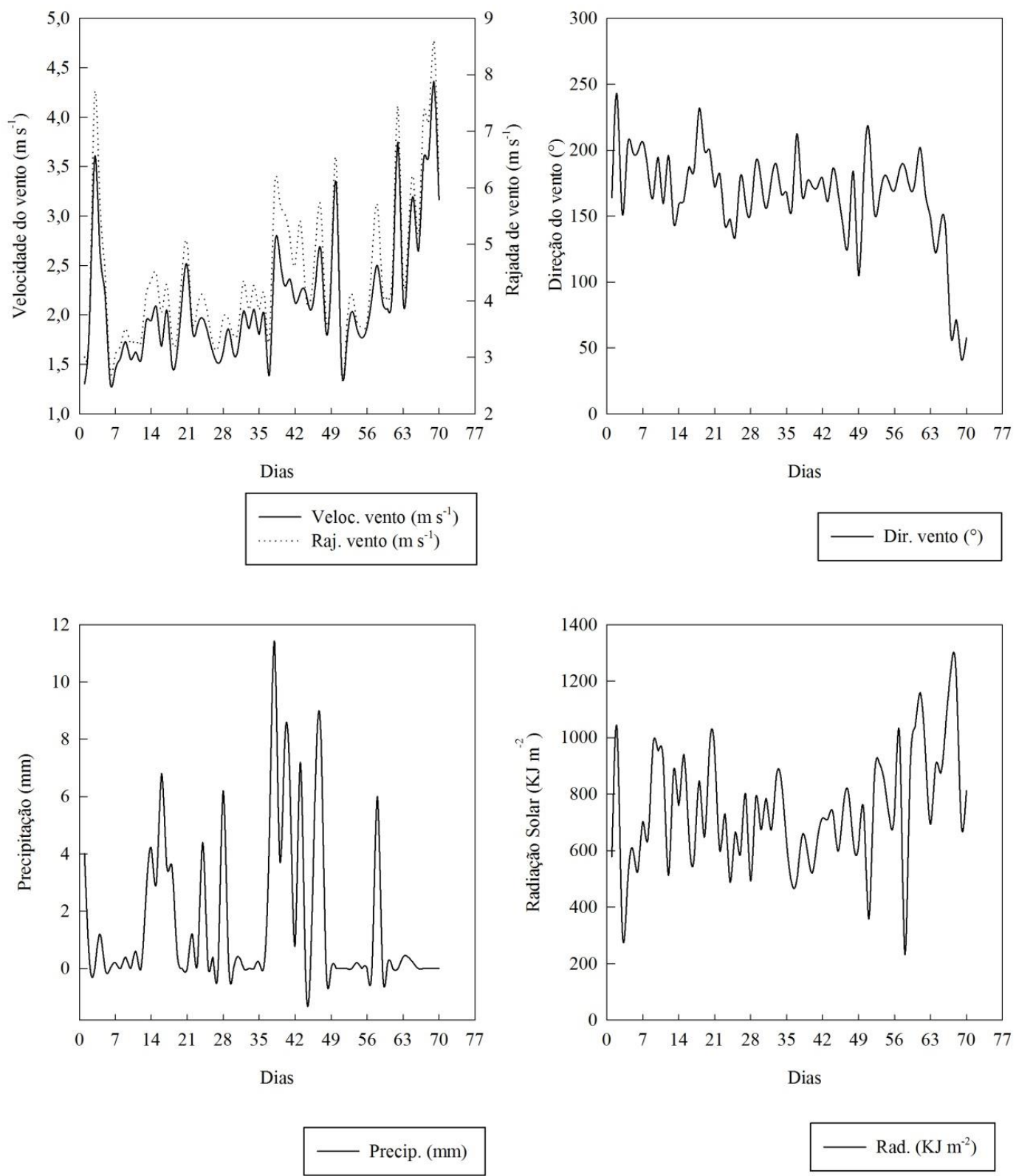

Na Figura 3, observa-se o crescimento e desenvolvimento da aveia-preta. Aos 70 dias, já apresentava em estádio de final de floração (BLEIHOLDER et al., 2001). Mesmo com o nas condições edafoclimáticas da região norte do ES. Este, possivelmente, foi beneficiado pela textura, visto que não há nenhum impedimento para o sistema radicular da planta. 
Figura 3 - O crescimento e desenvolvimento da aveia-preta aos 49, 56, 63 e 70 dias após a semeadura (DAS).

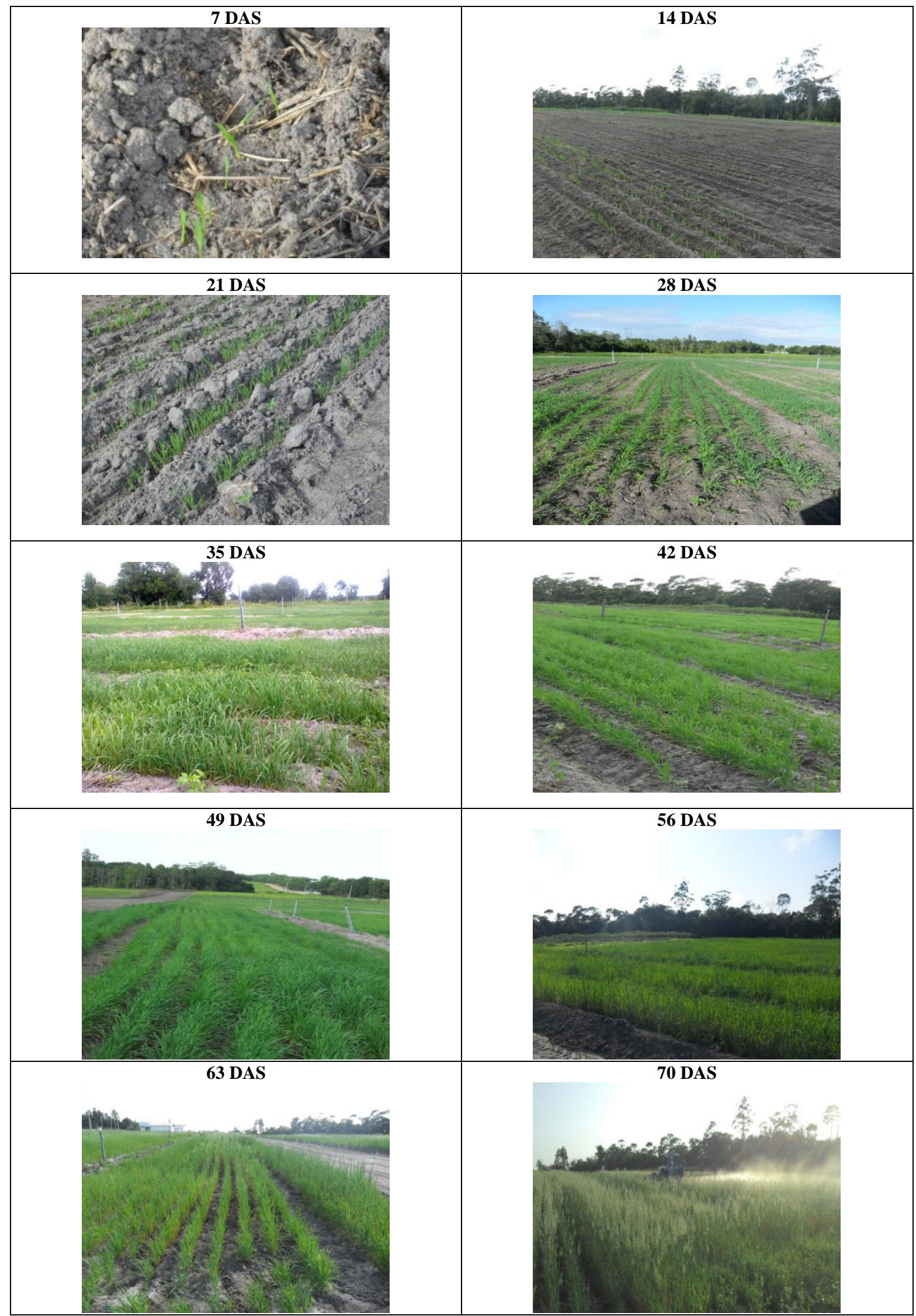




\section{CONCLUSÕES}

A aveia-preta desenvolve bem nas condições edafoclimáticas da região de São Mateus-ES, principalmente, sobre um Argiloso Amarelo de textura do tipo areia-franca.

\section{AGRADECIMENTOS}

A Fundação de Amparo à Pesquisa do Espírito Santo (FAPES) pelo recurso financeiro e a Coordenação de Aperfeiçoamento de Pessoal de Nível Superior (CAPES), pela concessão da bolsa de Programa de Fixação de Doutores no Estado do Espírito Santo (PROFIX).

\section{REFERÊNCIAS BIBLIOGRÁFICAS}

BLEIHOLDER, H.; WEBER, E.; HESS, M.; WICKE, H.; VAN DEN BOOM, T.; LANCASHIRE, P.; BUHR, L.; HACK, H.; KLOSE, R.; STAUSS, R. Growth Stages of Mono- and Dicotyledonous Plants, BBCH Monograph. Federal Biological Research Centre for Agriculture and Forestry: Berlin/Braunschweig, Germany, 2001; p. 158.

BURLE, M. L.; CARVALHO, A. M. de; AMABILE, R. F.;PEREIRA, J. Caracterização das espécies de adubo verde. In: CARVALHO, A. M. de; AMABILE, R. F. EMBRAPA CERRADOS. Cerrado: adubação verde. Planaltina, DF: Embrapa Cerrados, 2006. 369p.

CALEGARI, A.; ALCÂNTARA, P. B.; MIYASAKA, S.; AMADO, T. J. C. Caracterização das principais espécies de adubo verde. In: CALEGARI, A.; MONDARDO, A.; BULISANI, E. A.; WILDNER, L. do P.; COSTA, M. B. B. da; ALCÂNTARA, P. B.; MIYASAKA, S.; AMADO, T. J. C. Adubação verde no sul do Brasil. 2. ed. Rio de Janeiro: AS-PTA, 1993. p.207-327.

DAY, P. R. Particle fractionation and particle-size analysis. In: Black, C. A. (Ed.). Methods of soil analysis. 2nd ed. Madison: American Society of Agronomy, 1965. v.1, p.545-566.

$\begin{array}{cccc}\text { EMPRESA } & \text { BRASILEIRA } & \text { DE } & \text { PESQUISA } \\ \text { AGROPECUÁRIA. Centro Nacional de Pesquisa de }\end{array}$

Solos. Sistema brasileiro de classificação de solos. 3. ed. Rio de Janeiro, 2013. 353p.

EMPRESA BRASILEIRA DE PESQUISA AGROPECUÁRIA. Serviço Nacional de Levantamento e Conservação do Solo. Manual de métodos de análises do solo. Rio de Janeiro: Ministério da Agricultura, 1997. $212 \mathrm{p}$.

FEROLlA, F. S.; VÁSQUEZ, H. M.; SILVA, J. F. C.; VIANA, A. P.; DOMINGUES, F. N.; AGUIAR, R. S.. Produção de matéria seca, composição da massa de forragem e relação lâmina foliar/caule + bainha de aveiapreta e triticale nos sistemas de corte e de pastejo. Revista Brasileira de Zootecnia, v. 36, n. 5, p. 15121517, 2007 (supl.).

MISSOURI BOTANICAL GARDEN. Tropicos®. Disponível em: http://www.tropicos.org/NameSearch.aspx. Acesso em: 20 de outubro 2015.

PEEL, M. C.; FINLAYSON, B. L. \& MCMAHON, T. A. Updated world map of the Köppen-Geiger climate classification. Hydrology Earth System Sciences, v. 11, n. 5, p. 1633-1644, 2007.

SCHUCH, L. O. B.; NEDEL, J. L.; ASSIS, F. N. de; MAIA, M. DE S. Crescimento em laboratório de plântulas de aveia-preta (Avena Strigosa Schreb.) em função do vigor das sementes. Pesquisa Brasileira de Sementes, v. 21, n. 1, p. 229-234, 1999.

SOUZA, D. M. G.; LOBATO, E. Calagem e adubação para culturas anuais e semiperenes. In: SOUZA, D. M. G.; LOBATO, E. Cerrado: correção do solo e adubação. 2. ed. Brasília, DF: Embrapa Informação Tecnológica, 2004. 416 p.

WUTKE, E. B.; CALEGARI, A.; WILDNER, L. do P. Espécies de adubos verdes e plantas de cobertura e recomendação para seu uso. In: LIMA FILHO, O. F.; AMBROSANO, E. J.; ROSSI, F.; CARLOS, J. A. D. Adubação verde e plantas de cobertura no Brasil: Fundamentos e Prática. Brasília, DF: Embrapa, 2014. v.1. 507p. 